\title{
Screening and the quantitative $\pi$-model description of the optical spectra and polarizations of phenyl based oligomers
}

\author{
C. W. M. Castleton ${ }^{\text {a) }}$ \\ European Synchrotron Radiation Facility, B.P. 220, F-38043 Grenoble Cédex, France \\ W. Barford \\ Department of Physics and Astronomy, The University of Sheffield, Sheffield, S3 7RH, United Kingdom
}

(Received 3 July 2001; accepted 8 May 2002)

\begin{abstract}
The long standing problem of the inability of many semiempirical models to correctly predict the polarization of the higher dipole allowed optical transitions of phenyl based $\pi$-conjugated polymers and molecules is examined and related to the issue of internal and external screening of $\pi-\pi$ electron Coulomb interactions within the molecules. Following a review of previous theoretical and experimental work, $\pi$ electron only the Complete Neglect of Differential Overlap (CNDO) model is presented which, for the first time, is able to predict accurately the energies and symmetries of all the observed optical transitions of benzene, biphenyl and trans-stilbene, up to $\sim 8-10 \mathrm{eV}$. In so doing, it is demonstrated that the problem with previous calculations was the noninclusion of screening from outside the $\pi$ electron system itself. By fitting separately the spectra in hydrocarbon based condensed phases, in the gas phase and in solid rare gas matrices, and comparing the resulting model parameters, we show that, while the effects of screening from the environment are certainly noticeable, the most important spectral features - in particular the ordering of dipole allowed transitions - come from effective screening by the $\sigma$ electrons. We find that both of these effects can be adequately accounted for within a $\pi$ electron only model by using a dielectric constant and appropriate parameter renormalization. (C) 2002 American Institute of Physics.
\end{abstract}

[DOI: $10.1063 / 1.1489994]$

\section{INTRODUCTION}

The low lying electronic states and optical transitions of $\pi$-conjugated polymers, such as poly-(para-phenylene) and poly-(para-phenylene-vinylene) and their substituted analogs, are of great interest, both in terms of their basic physics and because of their technological applications. However, their theoretical description remains a formidable challenge, since Hilbert spaces are immense, even for the shortest oligomers. Any theoretical description thus requires approximations, usually either (i) exact solutions to approximate models (semiempirical modeling) or (ii) approximate solutions to quasiexact models ( $a b$ initio). In the latter very few degrees of freedom are omitted, so calculations are very large, usually relying on, for example, restricted bases, local exchange, and/or other types of mean field approximations. These occasionally fail, and they cannot easily tell us which factors are the most important. Semiempirical models usually leave out many degrees of freedom and interactions, hoping to capture the essential physics in what remains. In this way they help us to identify which properties and interactions are the most fundamental. If they successfully describe experimental reality then that which was omitted can reasonably be considered unimportant.

Unfortunately, in the area of $\pi$-conjugated polymers and molecules, these models have not always been very effective.

\footnotetext{
a) Present address: Fysik IV, Box 530, Uppsala Universitet, 75121 Uppsala, Sweden.
}

Models involving the $\pi$ electrons alone and using Coulomb type potentials never describe more than the first optically allowed transition correctly. ${ }^{1-4}$ The best that can be done was recently presented by the current authors, ${ }^{5,6}$ who reoptimized the Pariser-Parr-Pople (PPP) model ${ }^{7,8}$ to describe the lowest singlets and triplets of benzene, biphenyl, and trans-stilbene. The main problem is that, no matter what parameter set is used, the second optically allowed transition is found to be polarized parallel to the long axis of the molecule, and the third perpendicular, while experimentally they come in the opposite order. This is also true of calculations on longer oligomers and the polymers themselves. (In this discussion we omit any weak particle-hole forbidden transitions, referring for the moment only to the strong absorption bands.) Semiempirical models that directly include the $\sigma$ electrons sometimes work, ${ }^{9,10}$ and sometimes do not. ${ }^{2,11}$ This is probably due partly to poor parameterization, and partly to incomplete treatment of correlation effects. Calculations are rarely reported beyond the double configuration interaction (DCI) level, despite the demonstration by Schulten et al. ${ }^{12}$ that this is not always sufficient. On the other hand, calculations by Moore et al. ${ }^{13,14}$ indicate that including the environment but not the $\sigma$ electrons can, for example, give good exciton binding energies.

So what is missing from the $\pi$ electron only calculations? It has been suggested ${ }^{15}$ that the most important missing element is the environment, which should (at the least) screen the Coulomb interactions between the $\pi$ electrons. ${ }^{13,14}$ Clearly the $\sigma$ electrons are also missing, and this may be 
more important. They make several different contributions. It is accepted that the low lying spectra should be comprised of $\pi-\pi^{*}$ transitions, but above some particular energy $\pi-\sigma^{*}$ and $\sigma-\pi^{*}$ type transitions should become important, as well as ionization and transitions to Rydberg states. Above this energy (about 6-8 eV) we do not expect a $\pi$ only model to work, but below this it is surprising to need to include the $\sigma$ electrons directly. There is, however, a second role played by the $\sigma$ electrons, even at lower energies. This can be thought of, roughly, as screening. In the full Hamiltonian, with all differential overlap, the wave functions corresponding to the states occupied by the $\sigma$ electrons will change a little in response to the motion of the $\pi$ electrons, even when $\sigma-\pi^{*}$ type transitions do not occur; hence, they contribute to the screening of the Coulomb interactions between the $\pi$ electrons. In the complete Hamiltonian for the system these changes are contained within differential overlap matrix elements, and are therefore lost in a complete neglect of differential overlap (CNDO) approximation and hence in the PPP model. At the same time we should, in principle, Wannier orthogonalize both the $\pi$ electron states included and the $\sigma$ electron states omitted, so speaking of screening within the resulting model is formally not quite correct. However, the Wannier orthogonalization is normally taken "as read," and the diagonal overlap matrix elements (which become the parameters of the semiempirical CNDO model), are renormalized to best make up for the omitted off-diagonal terms, and it is in this sense that the word "screening" may be used. Previous CNDO calculations have not done this well, and the main aim of this paper is to do it better.

Sceening in $\pi$ electron systems comes from three sources: (1) from the other $\pi$ electrons, (2) from the environment, (3) from the $\sigma$ electrons. As discussed by Moore et al. ${ }^{13,14}$ the sources of screening can also be classified by their time constants. In "fast" screening the charges doing the screening rearrange on a time scale shorter than those whose interactions are being screened; A classical dielectric. The screening charges can then be averaged over all of their configurations subject to a specific configuration of the charges being screened. Hence the screening can be described by a simple dielectric constant scaling down the Coulomb interactions. In "slow" screening the screening charges react more slowly than those being screened. The average over configurations is no longer possible, so a complete treatment must include the screening sources themselves.

Screening from other $\pi$ electrons is clearly in neither limit. We will use sparse matrix exact diagonalization techniques [Lanczos, equivalent to complete configuration interaction $(\mathrm{CCI})]$ so we will treat the $\pi$ electron screening exactly. (At the partial CI $\pi$ electron screening is only partially treated.) Since we thus solve the model exactly any failure is due directly to inadequate treatment of the environment or of the $\sigma$ electrons.

Environmental screening from a hydrocarbon solvent is also intermediate in rate, as shown by Moore et al. ${ }^{13,14} \mathrm{We}$ therefore cannot treat it completely without including solvent molecules, but this would make the Hilbert space too large. We do not yet know the screening rate for the $\sigma$ electrons, though either intermediate or fast seems perhaps most likely.
We will try to account for these two screening sources by including a dielectric constant $\epsilon$, in combination with additional renormalization of the other parameters of the model.

After reviewing previous experimental and theoretical spectra for these molecules in Sec. II and introducing our model in Sec. III we will in Sec. IV try to fit the spectra in the hydrocarbon condensed phases, where experimental data are more extensive. For comparison, we then continue to the vapor and noble gas matrix spectra, where environmental screening (should be) absent, before concluding in Sec. VI.

\section{REVIEW OF THE SPECTRA: THE ROLE OF THE ENVIRONMENT}

The first columns of Tables I-III give a summary of the experimental spectra of benzene, biphenyl, and stilbene. The full experimental data, with notes and references are given in an EPAPS deposit accompanying this paper. ${ }^{16}$ Since we show experimental and theoretical spectra on the same tables we list and label all of the states as per the condensed phase CCI spectra to be presented in Sec. IV. Hence the approximate particle-hole symmetry is used and for the ${ }^{1} B_{u}^{-}$states of stilbene we add labels $\|$ or $\perp$ for convenience, indicating polarization relative to the long molecular axis.

Carefull examination of the individual experimental values for each peak (see EPAPS deposit ${ }^{16}$ ) show that there is a distinct uniformity amongst the hydrocarbon condensed phase spectra. The measured energies of vertical transitions show only a rather small spread, usually about $0.01-0.05 \mathrm{eV}$, sometimes up to $0.1-0.2 \mathrm{eV}$. Peak broadening from phonons and other sources of uncertainty mean that, despite the number of decimal places often quoted, the location of vertical transitions is usually only certain to about $0.02-0.10 \mathrm{eV}$, depending upon the experiment. Moreover, the same transition in the same environment but different experiments can vary by $0.01-0.05 \mathrm{eV}$. The uncertainty in the vertical transition energies is then similar to the variation of those energies between different hydrocarbon based environments, but rather different to the variation with respect to other environments. This suggests that the screening effect of a hydrocarbon environment is roughly the same, irrespective of the hydrocarbon in question, so the spectra can be combined to form a single composite data set. Solvent shifts from the vapor are $\sim 0.1-0.5 \mathrm{eV}$ for these molecules, so the vapor spectra and also the spectra from dilute solid solutions in noble gases are distinct and must be considered separately. For each state we now find a narrow energy range within which the vertical transition may lie for a particular environment and it is these which are given in Tables I-III. (For some of the higher states, where only 1 or 2 experimental values exist, the ranges given in the tables are taken from peak broadening, etc., in the individual experiments.) In principle it would be preferable to keep to a single environment (e.g. pure crystals) but that would leave us with too few states to fit. A semiempirical fit needs to involve significantly more fitted states than model parameters, and is meaningless unless the symmetries and polarizations of those states are also fitted.

Looking at the spectra themselves, those of biphenyl and stilbene have much the same structure, as expected since 
they are very similar in form. Both have three optically allowed transitions below $\sim 6.5 \mathrm{eV}$, where pure $\pi-\pi^{*}$ transitions are expected, and three above. For both these are polarized in the order $\|\perp\| \| \perp \perp$. For stilbene the upper three have only been seen in an argon matrix, ${ }^{17}$ where the fourth and fifth transitions overlap to produce a single band, with the polarization changing abruptly about halfway up the broad rising edge. An additional weak (probably particlehole forbidden) transition lies below the first strong allowed transition for biphenyl. The equivalent for stilbene has not been identified, but probably contributes to the long tail of the first allowed band.

Between the second and third dipole allowed transitions at least one 2 photon transition has been seen for each molecule, with two more below the second allowed. In stilbene these latter lie above the first allowed, but for biphenyl they lie below. This means that biphenyl cannot electroluminesce while stilbene could.

There have been claims of additional, very weak, low lying ${ }^{1} A_{g}$ states for both molecules. ${ }^{18-20}$ In stilbene it would constitute a low shoulder to the much stronger $2{ }^{1} A_{g}^{+}$state above it, while in biphenyl it is part of the long tail of the $1^{1} B_{3 g}^{+}$state. However, for example, it is not seen in calculations for stilbene using either Multiconfigurational Second Order Perturbation Theory ${ }^{21}$ (CASPT2), the Zerner Intermediate Neglect of Differential Overlap (ZINDO) program $^{22}$ or the Time Dependent Density Functional Theory ${ }^{22}$ (TD-DFT), and for both molecules it happens to coincide with the energy of the first strong allowed transition.

For biphenyl two low lying triplets are known: $1{ }^{3} B_{1 u}^{+}$ and (probably) $1{ }^{3} B_{2 u}^{+}$though the symmetry is uncertain as it is known only from EELS. Only one such state is known for stilbene. $T_{0} \rightarrow T_{N}$ absorption in biphenyl gives a strong transition to a ${ }^{3} A_{g}$ state $3.35 \mathrm{eV}$ above $1{ }^{3} B_{1 u}^{+}$and a broad weak region with many distinct phonon peaks at lower energy. This is thought to contain two weak transitions: one of $B_{3 g}$ symmetry, with triplet-triplet $\mathrm{OO} \sim 1.81 \mathrm{eV}$, (vertical $\sim 0.4$ $\mathrm{eV}$ higher,) the other of $A_{g}$ symmetry and triplet-triplet $\mathrm{OO}$ around $2.55 \mathrm{eV}^{23,24}$ The $\mathrm{OO}$ and vertical transitions are closer together for the ${ }^{3} A_{g}$ states. We know nothing directly about the vertical energies for $S_{0} \rightarrow T_{N}$ transitions to these states, but estimates are given in Table II based upon the width of the phonon broadening of the $T_{0} \rightarrow T_{N}$ spectrum, and taking the origin of the $1^{3} B_{1 u}^{+}$state as $2.84 \mathrm{eV}$. For stilbene only a single $T_{0} \rightarrow T_{N}$ transition has been detected, about $3.3 \mathrm{eV}$ above $T_{0}$.

Finally, the spectra for benzene are shown in Table I. Three states are seen in one photon absorption: $1 B_{1 u}^{-}$(formally forbidden in truly planar benzene,) the strong $1{ }^{1} E_{1 u}^{-}$, and a very broadband peaked at $\sim 11 \mathrm{eV}^{25}$ The low lying $1{ }^{1} B_{2 u}^{+}$is known and a ${ }^{1} E_{2 g}$ transition lies at $\sim 7.6 \mathrm{eV}$ in the gas phase. ${ }^{26,27} S_{1} \rightarrow S_{n}$ absorption ${ }^{28}$ has a peak at $\sim 8.9-9.6$ $\mathrm{eV}$ and a very broad shoulder at $\sim 7.3-8.5 \mathrm{eV}$. A second peak at 9.8-10.4 eV may or may not exist since error bars are large. These should formally be ${ }^{1} E_{2 g}$ states in planar benzene. An additional low lying ${ }^{1} E_{2 g}$ state has been reported ${ }^{29}$ in rare gas matrices but not seen in other measurements. The first three triplets are known too, although the third only from EELS, hence its symmetry is uncertain. $T_{1} \rightarrow T_{n}$ absorption ${ }^{28}$ reveals two broad peaks, and a weak shoulder, all formally due to states of ${ }^{3} E_{2 g}$ symmetry.

Comparing these spectra with existing theoretical ones, (for example, see biphenyl results in Table IV) the best to date using the PPP model have the second and third dipole allowed transitions in the wrong order, (amongst other errors) for both stilbene ${ }^{6}$ and biphenyl. ${ }^{5}$ Nonetheless, that calculation did predict that the lowest singlet transition is dipole forbidden for biphenyl, but dipole allowed for stilbene. For benzene, with no long or short axis, the calculation came out very well. ${ }^{5}$ Other CNDO calculations do even worse for both biphenyl and stilbene. ${ }^{1,3,4}$ The exception is that of Gudipati et al. ${ }^{17}$ for stilbene. They use a form for the Coulomb interaction which interpolates between fast and slowly decaying functions, to calculate the spectrum in an argon matrix. They get the order of the strong transitions correct, though, for example, the third dipole allowed transition is much too low for that matrix. However, they only calculate and fit the one photon allowed singlet spectrum, and thus have about the same number of model parameters as experimental points to fit. All the same, it does indicate that the difficulties lie with the Coulomb interactions. Several Intermediate Neglect of Differential Overlap (INDO) calculations exist, ${ }^{30}$ but do not quote the polarizations of the states, so it is impossible to assess their accuracy, apart from the recent ZINDO calculation mentioned above. In addition to this, TD-DFT (Ref. 22) and CASPT2 (Refs. 21, 31, 32) calculations have been performed. The ZINDO and ab initio techniques are much more complex and demanding than CNDO calculations, but generally yield qualitatively correct results, as one might expect.

Examining the gas and rare gas matrix spectra, we see that, apart from the overall shift of the spectra, they are in fact very similar to those for the hydrocarbon condensed phases. If the role of the $\sigma$ electrons were not significant, then earlier $\pi$ electron only calculations, ${ }^{3-6}$ which ignored all screening apart from that resulting from the $\pi$ electrons themselves, should describe the spectra in the gas and rare gas matrix phases quite well, at least up to about $6 \mathrm{eV}$ or so. However, as noted above, they predict the (incorrect) order II $\| \perp$ for the first three dipole allowed transitions. This entails a large reorganization of the spectra of biphenyl and stilbene in the gas phases, with changes on the order of at least 1.5 $\mathrm{eV}$. In fact, the one photon spectrum for dilute stilbene in an argon matrix has exactly the same structure as that in the hydrocarbon phases. The order and intensities of the polarizations are maintained, and the phonon side bands are in the same places relative to one another. The only real difference is a solvent shift of roughly $0.2 \mathrm{eV}$, reflecting the weaker environmental screening in noble gas matrices, as compared to hydrocarbon based environments. The shift is fairly rigid, ranging from $\sim 0.1$ to $\sim 0.4 \mathrm{eV}$ for different states, much less than the roughly $1.5 \mathrm{eV}$ needed to reverse the second and third allowed transitions. The biphenyl vapor spectrum seems to be much the same; there are few changes apart from an overall blueshift relative to the condensed phases. Unfortunately, the peak broadening, due mostly to single bond rotation, is very great. The $2{ }^{1} B_{2 u}^{-}$and $2{ }^{1} B_{1 u}^{-}$merge, and it is not actually possible to tell which is higher and which is lower. Nonetheless, they certainly do not swap over com- 
pletely, as the $\pi$ electron only calculations would predict. They remain close together, and single bond rotation alone probably accounts for the apparent overlap.

This shows that environmental screening is probably not the most important factor in determining the qualitative structure of the excitation spectra of these molecules within a PPP type model. Obviously, the environment does contribute to the spectra, with solvent shifts of the order of tenths of $\mathrm{eVs}$, but the experimental evidence is that the most important effects (in terms of the ordering of the states) come from elsewhere. Calculations ignoring $\sigma$ electron screening suggest effects of the order of $\mathrm{eV}$ coming from this.

\section{THE MODEL}

The normal $\pi$ electron only CNDO model is the PPP (Ref. 7) model, which is our start point,

$$
\begin{aligned}
\mathcal{H}= & \sum_{\langle i, j\rangle, \sigma} t_{i j}\left[c_{i \sigma}^{\dagger} c_{j \sigma}+c_{j \sigma}^{\dagger} c_{i \sigma}\right]+\sum_{i} U_{i}\left(n_{i \uparrow}-\frac{1}{2}\right)\left(n_{i \downarrow}-\frac{1}{2}\right) \\
& +\frac{1}{2} \sum_{i \neq j} V_{i j}\left(n_{i}-1\right)\left(n_{j}-1\right),
\end{aligned}
$$

where $c_{i \sigma}^{\dagger}$ creates a $\pi$-electron with spin $\sigma$ on site $i . n_{i \sigma}$ $=c_{i \sigma}^{\dagger} c_{i \sigma}, n_{i}=n_{i \uparrow}+n_{i \downarrow}$, and \langle\rangle gives the sum over nearest neighbors. In the CNDO approximation we could in principle have $U_{i}, V_{i j}$, and $t_{i j}$ different for each $\{i, j\}$, and use them all as fitting parameters. In practice we restrict ourselves to $U_{i}=U \forall i$. For $V_{i j}$ we use the Ohno ${ }^{8}$ potential, but add a dielectric constant $\epsilon$,

$$
V_{i j}=\frac{U}{\epsilon \sqrt{1+\alpha r_{i j}^{2}}} .
$$

For $t_{i j}$ we take the exponential form,

$$
t_{i j}=t_{p} \cos \theta_{i, j} \exp \left(\Delta\left(1+\frac{r_{i j}}{r_{p}}\right)\right)
$$

with $r_{p}$ and $t_{p}$ being, respectively, bond lengths and hopping integrals inside the phenyl rings. $\theta_{i, j}$ is the rotation angle of the $\pi$ system around bond $(i, j)$. This Hamiltonian is the spatial symmetry of the molecule, namely, $D_{6 h}$ for benzene, $D_{2 h}$ for biphenyl, and $C_{2 h}$ for stilbene, assuming strict planar geometries. Also conserved are preserves particle-hole symmetry and SU(2) spin symmetry, though we use only the conservation of $S^{z}$, operating in the $S^{z}=0$ subspace and using the "spin-flip" symmetry $\uparrow \Leftrightarrow \downarrow$ to divide the Hilbert space into symmetric $\left({ }^{3}\right)$ and antisymmetric $\left({ }^{1}\right)$-sectors.

With $\epsilon=1.0$ this is the model the authors optimized previously, ${ }^{5,6}$ the failures of which were discussed above. With $\epsilon \geqslant 1.0$ it has also been used previously, ${ }^{15}$ but the parameter set used was poor, and only SCI calculations were done. Performing exact (CCI) calculations using the same parameters we find that the spectra are rather badly described, with, for example, dipole forbidden states below ${ }^{1} B_{u}^{-}$for stilbene.

The value of $U$ is traditionally taken as $11.13 \mathrm{eV},{ }^{3,4}$ following Hinze and Jaffe $\mathrm{e}^{33}$ in 1962. This value was obtained as the difference between the ionization energy $(I)$ and electron affinity $(A)$ of an isolated $s p^{2}$ hybridized carbon atom. The assumption is that the process (0) of moving a $\pi$ electron from one atom to another (which already contains a $\pi$ electron) is equivalent to the sum of two processes: (1) the removal to infinity of an electron (ionization energy) and (2) bringing an electron from infinity (electron affinity) placing it on an already occupied atom. To a first approximation this is correct. However, process (2) ignores the presence of the hole left by (1). Also, the relaxation processes are assumed to have the same energy for $(1)+(2)$ as for (0) and the atomic core potential felt by the electron which moves is assumed to be the same on both atoms, irrespective of screening from other electrons on the atom. These are all significant effects. In addition, isolated $s p^{2}$ hybridized atoms do not exist, so Hinze and Jaffé started from the values of $I$ and $A$ for a spherically symmetric atom (then known only to $\pm 0.3 \mathrm{eV}$ ) and estimated the values for an $s p^{2}$ hybridized atom by summing over the relevant Slater integrals and fitting the free parameters to atomic spectra. Computational facilities being rather limited, they had to strongly approximate most of the integrals, and were unable to use configuration interactions fully. It is therefore very unlikely that the value quoted is nearly as accurate as the four significant figures often used.

The intraphenyl nearest neighbor hopping parameter $t_{p}$ is usually taken ${ }^{3,4}$ as $\sim 2.4 \mathrm{eV}$, loosely justified on the basis of estimates of the $t_{p} / U$ ratio, and early fits of the first few states of the molecules. ${ }^{3,34-36}$ The single and double bond hopping parameters $t_{s}$ and $t_{d}$ are usually thought $\mathrm{t}^{3,12,34}$ to lie around 2.2 and $2.6 \mathrm{eV}$, so $\Delta \sim 1.0 \longrightarrow 3.0$ is probably reasonable.

In practise these values have long been known to give poor results. Larger $t_{p}$ and smaller $U$ are normally required. For $\epsilon=1.0$ we found the optimal fit in hydrocarbon environments ${ }^{5,6}$ to be $U=10.06 \mathrm{eV}, t_{p}=2.539 \mathrm{eV}$, and $\delta=1.1422 \mathrm{eV}$, where we used the linearized form,

$$
t_{i j}=t_{p}\left(1+\delta\left(r_{p}-r_{i j}\right)\right)
$$

in place of Eq. (3).

Our approach in this paper is simply to vary the parameters in the model to obtain the best fit to the composite experimental spectra. For each parameter set, each energy is converged to better than 1 in $10^{8}$. A mean relative error compared to experiment, $\Gamma$, is calculated, and this is minimized with respect to the parameters. We use

$$
\Gamma=\frac{100 \%}{n} \sum_{n} \frac{\left\|E_{n}^{e \prime}-E_{n}^{c}\right\|}{E_{n}^{e \prime}+E_{n}^{c}},
$$

where the sum is over all of the states included in the fit, $E_{n}^{c}$ and $E_{n}^{e \prime}$ being the calculated and experimental energies. $E_{n}^{e \prime}$ is either the upper $E_{n}^{e+}$ or lower $E_{n}^{e-}$ limit of the experimentally acceptable ranges given in Tables I-III. If $E_{n}^{c}>E_{n}^{e+}$, then $E_{n}^{e \prime}=E_{n}^{e+}$, and if $E_{n}^{c}<E_{n}^{e-}$, then $E_{n}^{e \prime}=E_{n}^{e-}$. Otherwise, $E_{n}^{e \prime}=E_{n}^{c}$, i.e., we consider the error for a particular calculated energy to be zero if it lies within the fitting range. This error function is a little unusual, but is chosen to avoid biasing the fit. If we were to fit completely to, say $\frac{1}{2}\left(E_{n}^{e+}\right.$ $\left.+E_{n}^{e-}\right)$, we would end up overemphasizing the importance of the states that are least well known experimentally, at the cost of less accurately fitting the better known energies. $\Gamma=0 \%$ if all the calculated energies in the fit lie within the 
TABLE I. Experimental and theoretical spectra for benzene. Theoretical spectra are given at least up to $10 \mathrm{eV}$. The singlet state around $10 \mathrm{eV}$ should formally (assuming absolute planarity) be of $E_{2 g}$ symmetry, but best matches our $3{ }^{1} A_{1 g}^{+}$.

\begin{tabular}{|c|c|c|c|c|c|c|c|c|c|c|}
\hline \multirow[b]{3}{*}{ State } & \multicolumn{6}{|c|}{ Experimental spectra } & \multicolumn{4}{|c|}{ PPP spectra } \\
\hline & \multirow[b]{2}{*}{ EI } & \multirow{2}{*}{$\begin{array}{c}\text { Condensed } \\
\text { phases }\end{array}$} & \multicolumn{3}{|c|}{ Rare gas matrices } & \multirow{2}{*}{$\begin{array}{c}\text { Gas } \\
\text { phase }\end{array}$} & \multicolumn{2}{|c|}{ Condensed } & \multicolumn{2}{|c|}{ Gas } \\
\hline & & & Xenon & Krypton & Argon & & $E_{n}$ & $O_{n}$ & $E_{n}$ & $O_{n}$ \\
\hline \multicolumn{11}{|c|}{ Singlet spectrum } \\
\hline $1{ }^{1} B_{2 u}^{+}$ & vw & $4.66-5.01$ & $>4.74_{O}$ & & $4.89-5.34$ & $4.80-5.00$ & 4.929 & & 4.759 & \\
\hline$?{ }^{1} E_{2 g}$ & $\mathrm{vw}$ & & $5.81-5.91$ & $5.81-5.91$ & & & & & & \\
\hline $1{ }^{1} B_{1 u}^{-}$ & & $5.82-6.13$ & $6.09-6.21$ & $6.08-6.90$ & $6.00-6.12$ & $6.19-6.31$ & 6.055 & & 6.298 & \\
\hline $1{ }^{1} E_{1 u}^{-}$ & $\mathrm{s}$ & $6.35-6.80$ & $>6.58_{O}$ & $6.67-6.90$ & $6.73-6.85$ & $6.93-6.98$ & 6.476 & 0.988 & 6.932 & 0.982 \\
\hline $1{ }^{1} E_{2 g}^{+}$ & & $7.30-8.45$ & & & & $7.62-7.66$ & 7.742 & & 7.546 & \\
\hline $2{ }^{1} E_{2 g}^{-g}$ & & $8.95-9.55$ & & & & & 8.781 & & 9.100 & \\
\hline $2{ }^{1} A_{1 g}^{+}$ & & & & & & & 8.954 & & 8.582 & \\
\hline$?{ }^{1} E_{2 g}$ & & $9.85-10.20$ & & & & & & & & \\
\hline $3{ }^{1} A_{1 g}^{+}$ & & $9.85-10.20^{*}$ & & & & & 9.888 & & 9.796 & \\
\hline $1{ }^{1} A_{2 g}^{-}$ & & & & & & & 10.517 & & 10.594 & \\
\hline $2{ }^{1} E_{1 u}^{+}$ & & $9.2 \rightarrow>10.8$ & & & & & 10.836 & & 10.285 & \\
\hline \multicolumn{11}{|c|}{ Triplet spectrum } \\
\hline $1{ }^{3} B_{1 u}^{+}$ & & $3.75-4.05$ & $>3.66_{O}$ & $3.78-4.13$ & $>3.67_{O}$ & $3.85-4.00$ & 4.276 & & 4.000 & \\
\hline $1{ }^{3} E_{1 u}^{+}$ & & $4.62-4.68$ & $>4.61_{O}$ & $>4.67_{O}$ & & $4.70-4.80$ & 4.849 & & 4.747 & \\
\hline $1{ }^{3} B_{2 u}^{-}$ & & & & & & $5.55-5.65$ & 5.591 & & 5.834 & \\
\hline $1{ }^{3} E_{2 g}^{+}$ & $\mathrm{w}$ & $7.2-8.2$ & & & & & 6.942 & & 6.664 & \\
\hline $2{ }^{3} E_{2 g}^{-g}$ & $\mathrm{~s}$ & $8.6-9.2$ & & & & & 8.635 & 0.132 & 8.969 & 0.098 \\
\hline $2{ }^{3} B_{1 u}^{+}$ & & & & & & & 10.103 & & 9.801 & \\
\hline $4{ }^{3} E_{2 g}^{-}$ & & & & & & & 11.139 & 0.864 & 11.445 & 0.896 \\
\hline
\end{tabular}

${ }^{\mathrm{a}} \mathrm{All}$ states are labeled according to the condensed phase PPP results. All energies $\left(E_{n}\right)$ are in $\mathrm{eV}$, and are complete up to the horizontal line indicated. For more complete spectra see the EPAPS deposit (Ref. 16). Oscillator strengths $\left(O_{n}\right.$, or $O_{\|}$along the long molecular axis, $O_{\perp}$ along the short,) are always given relative to the lowest lying singlet or triplet. The experimental intensities (EI) given are intended as a rough guide indication. The following abbreviations are also used: Subscript $O$ indicates an OO transition (as identified in the papers quoted. The are included here only when the transition peak is not known.)? indicates an experimental state that we do not find within the theory. $\mathrm{vw}=$ very weak. $\mathrm{w}=$ weak. $\mathrm{m}=$ medium. $\mathrm{s}=$ strong.

experimental uncertainty. For an individual state, an error of a percent corresponds to being outside the experimental range by $\sim 0.1 \mathrm{eV}$, so is reasonable. Averaged over all states, on the other hand, $\Gamma$ can be fairly small, even when the spectra are essentially wrong. Our previous spectrum for stilbene, for example, which has the second and third dipole allowed transitions completely reversed, still has $\Gamma=2 \%$. Hence care must be taken when comparing different fits. For a fit to a spectrum of, say, 5-8 states, we would look for a value of $\Gamma=0.1 \%-1.0 \%$ or less.

We also calculate oscillator strengths along the long and short axes for biphenyl and stilbene. These are given by the projection onto the relevant axis of the square of the E1 dipole operator. For axis $x$, this is given for state $n$ by

$$
O_{n}^{x}=\varphi_{n}\left\langle\Psi_{n}\left|\sum_{i} x_{i} n_{i}\right| \Psi_{0}\right\rangle^{2},
$$

where the normalization is

$$
\varphi_{n}=\frac{\left(E_{n}-E_{0}\right)}{\left\langle\Psi_{0}\left|\Sigma_{i, j, \sigma}\left(x_{i}-x_{j}\right)^{2} c_{i \sigma}^{\dagger} c_{j \sigma}\right| \Psi_{0}\right\rangle} .
$$

The $O_{n}^{x}$ obey the sum rule $\Sigma_{n} O_{n}^{x}=1$.

\section{CALCULATING THE HYDROCARBON MATRIX/SOLUTION SPECTRA}

Since the problems with earlier calculations are most apparent in the polarization directions of the dipole allowed transitions, we start by fitting the spectrum for biphenyl, not benzene, and then proceed to that of stilbene before returning to benzene. We use the geometries shown in Fig. 1, but with all bond rotation angles $\left(\theta, \theta_{1}, \theta_{2}\right.$, and $\left.\phi\right)$ set to zero. These are taken from x-ray scattering data for bipheny $1^{37,38}$ and stilbene. $^{39-41}$ For simplicity we use planar geometries throughout, though we note here that this is not wholly appropriate in liquid phases.

\section{A. Fitting the spectra}

We start by calculating the spectra of biphenyl and stilbene over very wide ranges for $U, t_{p}, \Delta$, and $\epsilon$, from the unphysically small to the unphysically large, and all between. No parameter range with $\epsilon=1.0$ is found for which the dipole allowed transitions occur in the correct order. $\epsilon>1.0$ is definitely required. Considering the biphenyl states $1{ }^{1} B_{3 g}^{+}, 1{ }^{1} B_{2 u}^{+}, 1{ }^{1} B_{1 u}^{-}, 2{ }^{1} B_{2 u}^{-}, 2{ }^{1} B_{1 u}^{-}$, and $1{ }^{3} B_{1 u}^{+}$, we find that a "perfect" fit (i.e., with $\Gamma=0.0 \%$ ) occurs around $U=7.0 \mathrm{eV}, t_{p}=2.6 \mathrm{eV}, \Delta=2.0-3.0, \epsilon=1.5$.

The ${ }^{1} A_{g}^{+}$states have been omitted here since there may or may not be an additional one at $4.45-4.84 \mathrm{eV}$. The fit obtained puts $2{ }^{1} A_{g}^{+}$at $\sim 5.96 \mathrm{eV}$. This matches the experimental ${ }^{1} A_{g}$ state at around $6 \mathrm{eV}$, but is too high for the additional state. The first particle-hole forbidden ${ }^{1} A_{g}$ state lies at $6.131 \mathrm{eV}$ so this is not the additional state either. Redoing the fit but adding 2 and $3{ }^{1} A_{g}^{+}$at $4.45-4.84 \mathrm{eV}$ and $5.64-6.14 \mathrm{eV}$, to try to force the existence of the state, we find $\Gamma=1.00 \%$, but obtain unphysical values for $\Delta$ and $\epsilon$. 
TABLE II. Experimental and theoretical spectra for bipheynl: All theoretical states are included up to at least 7 eV. See footnote "a" in Table I.

\begin{tabular}{|c|c|c|c|c|c|c|c|c|}
\hline \multirow[b]{3}{*}{ State } & \multicolumn{4}{|c|}{ Experimental spectra } & \multicolumn{4}{|c|}{ PPP spectra } \\
\hline & \multirow[b]{2}{*}{ EI } & \multirow{2}{*}{$\begin{array}{l}\text { Condensed } \\
\text { phases }\end{array}$} & \multirow{2}{*}{$\begin{array}{l}\text { Neon } \\
\text { matrix }\end{array}$} & \multirow{2}{*}{$\begin{array}{c}\text { Gas } \\
\text { Phase }\end{array}$} & \multicolumn{2}{|c|}{ Condensed } & \multicolumn{2}{|c|}{ Gas } \\
\hline & & & & & $E_{n}$ & $O_{n}$ & $E_{n}$ & $O_{n}$ \\
\hline \multicolumn{9}{|c|}{ Singlet spectrum } \\
\hline $1^{1} B_{2 u}^{+}$ & $\mathrm{vw}$ & $4.50-4.65$ & & $4.60 \pm 0.20$ & 4.600 & & 4.503 & \\
\hline $1{ }^{1} B_{3 g}^{+}$ & $\mathrm{vw}$ & $4.10-4.70$ & $>4.14_{O}$ & & 4.625 & & 4.520 & \\
\hline$?{ }^{1} A_{g}$ & vw & $4.45-4.85$ & & & & & & \\
\hline $1{ }^{1} B_{1 u}^{-}$ & $\mathrm{m}$ & $4.75-5.05$ & & $5.21 \pm 0.15$ & 4.800 & 0.427 & 5.123 & 0.398 \\
\hline $2{ }^{1} B_{3 g}^{-}$ & & & & & 5.701 & & 6.187 & \\
\hline $2{ }^{1} B_{2 u}^{-g}$ & $\mathrm{~s}$ & $5.80-6.00$ & & $6.41 \pm 0.10$ & 5.939 & 0.530 & 6.368 & 0.450 \\
\hline $2{ }^{1} A_{g}^{+}$ & $\mathrm{s}$ & $5.64-6.14$ & & & 6.062 & & 6.082 & \\
\hline $3{ }^{1} A_{g}^{-}$ & & & & & 6.067 & & 6.309 & \\
\hline $2{ }^{1} B_{1 u}^{-g}$ & $\mathrm{~s}$ & $6.12-6.19$ & & $6.41 \pm 0.10$ & 6.178 & 0.493 & 6.502 & 0.501 \\
\hline $4{ }^{1} A_{g}^{-}$ & & & & & 6.678 & & 7.162 & \\
\hline $5{ }^{1} A_{g}^{+}$ & & & & & 6.685 & & 7.019 & \\
\hline $3{ }^{1} B_{3 g}^{+}$ & & & & & 6.755 & & 6.842 & \\
\hline $3{ }^{1} B_{2 u}^{+}$ & & & & & 6.767 & & 6.847 & \\
\hline $3{ }^{1} B_{1 u}^{-}$ & $\mathrm{w}$ & $6.7-7.1$ & & $7.10 \pm 0.15$ & 6.775 & 0.001 & 7.228 & 0.001 \\
\hline $4{ }^{1} B_{2 u}^{-}$ & s & $7.0-7.3$ & & $7.66 \pm 0.13$ & 7.353 & 0.427 & 7.735 & 0.363 \\
\hline $5^{1} B_{1 u}^{-}$ & $\mathrm{w}$ & $7.9-8.2$ & & $8.31 \pm 0.15$ & 7.793 & 0.059 & 8.158 & 0.075 \\
\hline $7{ }^{1} B_{1 u}^{-u}$ & & & & & 8.320 & 0.002 & 8.685 & 0.001 \\
\hline \multicolumn{9}{|c|}{ Triplet spectrum } \\
\hline $1{ }^{3} B_{1 u}^{+}$ & & $2.85-3.90$ & $>2.85_{O}$ & & 3.574 & & 3.435 & \\
\hline $1^{3} A_{g}^{+}$ & & & & & 4.400 & & 4.139 & \\
\hline $1{ }^{3} B_{2 u}^{+}$ & & $4.00-4.10$ & & & 4.530 & & 4.489 & \\
\hline $1{ }^{3} B_{3 g}^{+}$ & & & & & 4.532 & & 4.490 & \\
\hline $2{ }^{3} B_{1 u}^{+}$ & & & & & 4.636 & & 4.510 & \\
\hline $2{ }^{3} A_{g}^{+}$ & & & & & 4.896 & & 4.790 & \\
\hline $2{ }^{3} B_{2 u}^{-}$ & & & & & 5.160 & & 5.445 & \\
\hline $2{ }^{3} B_{3 g}^{-}$ & $\mathrm{w}$ & $\sim 4.9-5.3$ & & & 5.196 & 0.005 & 5.471 & 0.003 \\
\hline$?{ }^{3} A_{g} g$ & w & $\sim 5.3-5.6$ & & & & & & \\
\hline $3{ }^{3} B_{1 u}^{+}$ & & & & & 6.335 & & 6.148 & \\
\hline $3{ }^{3} B_{3 g}^{+}$ & & & & & 6.346 & & 6.239 & \\
\hline $3{ }^{3} B_{2 u}^{+}$ & & & & & 6.358 & & 6.245 & \\
\hline $3{ }^{3} A_{g}^{-}$ & $\mathrm{s}$ & $\sim 6.1-6.3$ & & & 6.530 & 0.419 & 6.900 & 0.382 \\
\hline $4{ }^{3} B_{1 u}^{+}$ & & & & & 6.695 & & 7.029 & \\
\hline $4{ }^{3} A_{g}^{-}$ & & & & & 6.785 & $2 \times 10^{-4}$ & 7.241 & $2 \times 10^{-5}$ \\
\hline $5{ }^{3} A_{g}^{+}$ & & & & & 7.077 & & 6.797 & \\
\hline $4{ }^{3} B_{3 g}^{-}$ & & & & & 7.089 & 0.005 & 7.457 & 0.003 \\
\hline $9^{3} B_{3 g}^{-}$ & & & & & 9.152 & 0.149 & 9.346 & 0.075 \\
\hline $10^{3} A_{g}^{-g}$ & & & & & 9.724 & $\sim 0.04$ & 10.03 & $\sim 0.2$ \\
\hline $12^{3} B_{3 g}^{-g}$ & & & & & 9.945 & $\sim 0.1$ & 10.17 & $\sim 0.1$ \\
\hline $12^{3} A_{g}^{-g}$ & & & & & 9.945 & $\sim 0.4$ & & \\
\hline
\end{tabular}

$2{ }^{1} A_{g}^{+}$still comes no lower than $\sim 5.7 \mathrm{eV}$. Similarly, we find no evidence for the state in stilbene, and so conclude that there is $n o$ additional low lying ${ }^{1} A_{g}$ state for either case. Any in plane twisting of phenyl units would reduce the true symmetry of either molecule to $C_{1}$, thus mixing $A$ and $B$ states. They would then become very slightly two-photon allowed. Since the spectra were taken in liquid solution this could account for the measured intensity. In all further calculations it is presumed that $2{ }^{1} A_{g}^{+}$lies around $6 \mathrm{eV}$ in biphenyl and around $4.4 \mathrm{eV}$ in stilbene.

Now knowing the location of $2{ }^{1} A_{g}^{+}$we refine the biphenyl spectrum fit, fitting $1{ }^{1} B_{3 g}^{+}, 1{ }^{1} B_{2 u}^{+}, 1{ }^{1} B_{1 u}^{-}, 2{ }^{1} B_{2 u}^{-}$, $2{ }^{1} A_{g}^{+}, 2{ }^{1} B_{1 u}^{-}$, and $1{ }^{3} B_{1 u}^{+}$. We can still fit the spectrum with $\Gamma=0.0 \%$, obtaining a fairly narrow region of parameter space within which we fit the spectrum of stilbene. The Hilbert space for stilbene is very large indeed, so we focus first on the observed singlet spectrum: the states $1{ }^{1} B_{u}^{-}(\|)$, $3{ }^{1} B_{u}^{-}(\perp), 5{ }^{1} B_{u}^{-}(\|), 2{ }^{1} A_{g}^{+}$, and $3{ }^{1} A_{g}^{+}$, plus lower bounds for $7{ }^{1} B_{u}^{-}$and $6{ }^{1} A_{g}^{+}$. (Upturns near the edge of the energy range of 1 and 2 photon absorption experiments ${ }^{20}$ indicate the OO transitions, but do not give the vertical transitions.) The labels $(\|)$ and $(\perp)$ for the ${ }^{1} B_{u}^{-}$states are obtained by calculating oscillator strengths along the long and short molecular axes. The weak transition to an ${ }^{1} A_{g}$ state around 5.6 $\mathrm{eV}$ is omitted since initial calculations showed it to be due to the $5{ }^{1} A_{g}^{-}$state rather than a ${ }^{1} A_{g}^{+}$state. 
TABLE III. Experimental and theoretical spectra for stilbene: theoretical spectra are given for the condensed phases, and for the gas phase parameters with bond rotation and planar for comparison to the argon gas matrix spectrum, and are complete up to at least $7 \mathrm{eV}$. In addition results for the states 9 $\rightarrow 16{ }^{1} B_{u}^{-}$are converged to at least the accuracy quoted. See footnote "a" in Table I.

\begin{tabular}{|c|c|c|c|c|c|c|c|c|c|c|c|c|c|}
\hline \multirow[b]{3}{*}{ State } & \multicolumn{4}{|c|}{ Experimental spectra } & \multicolumn{9}{|c|}{ PPP spectra } \\
\hline & \multirow[b]{2}{*}{ EI } & \multirow{2}{*}{$\begin{array}{l}\text { Cond. } \\
\text { phases }\end{array}$} & \multirow{2}{*}{$\begin{array}{l}\text { Argon } \\
\text { matrix }\end{array}$} & \multirow{2}{*}{$\begin{array}{c}\text { Gas } \\
\text { phase }\end{array}$} & \multicolumn{3}{|c|}{ Condensed phases } & \multicolumn{3}{|c|}{$\phi=\theta_{1}=\theta_{2}=0^{\circ}$} & \multicolumn{3}{|c|}{$\phi=7^{\circ}, \theta_{1}=-\theta_{2}=30^{\circ}$} \\
\hline & & & & & $E_{n}$ & $O_{\|}$ & $O_{\perp}$ & $E_{n}$ & $O_{\|}$ & $O_{\perp}$ & $E_{n}$ & $O_{\|}$ & $O_{\perp}$ \\
\hline \multicolumn{14}{|c|}{ Singlet Spectrum } \\
\hline $1{ }^{1} B_{u}^{-}(\|)$ & $\mathrm{m}$ & $3.98-4.04$ & $4.05-4.09$ & $>4.00_{O}$ & 3.992 & 0.496 & 0.027 & 4.360 & 0.491 & 0.026 & 4.371 & 0.485 & 0.038 \\
\hline $2{ }^{1} B_{u}^{+}$ & & & & & 4.383 & & & 4.323 & & & 4.333 & & \\
\hline$?{ }^{1} A_{g}$ & $\mathrm{vw}$ & $4.10-4.14$ & & & & & & & & & & & \\
\hline $2{ }^{1} A_{g}^{+}$ & $\mathrm{m}$ & $4.38-4.46$ & & & 4.386 & & & 4.325 & & & 4.333 & & \\
\hline $3{ }^{1} A_{g}^{+}$ & $\mathrm{s}$ & $4.99-5.07$ & & & 5.063 & & & 5.054 & & & 5.059 & & \\
\hline $4{ }^{1} A_{g}^{-}$ & & & & & 5.362 & & & 5.806 & & & 5.828 & & \\
\hline $3{ }^{1} B_{u}^{-}(\perp)$ & $\mathrm{w}$ & $5.35-5.43$ & $5.55-5.76$ & & 5.412 & $8 \times 10^{-4}$ & 0.286 & 5.862 & $5 \times 10^{-4}$ & 0.276 & 5.854 & 0.001 & 0.266 \\
\hline $5{ }^{1} A_{g}^{-}$ & $\mathrm{m}$ & $5.60-5.68$ & & & 5.535 & & & 5.870 & & & 5.870 & & \\
\hline $4{ }^{1} B_{u}^{+}$ & & & & & 5.816 & & & 5.756 & & & 5.731 & & \\
\hline $5{ }^{1} B_{u}^{-}(\|)$ & $\mathrm{s}$ & $6.06-6.16$ & $6.3-6.4$ & & 6.138 & 0.216 & 0.006 & 6.416 & 0.198 & 0.009 & 6.411 & 0.190 & 0.012 \\
\hline $6{ }^{1} A_{g}^{+}$ & $\mathrm{s}$ & $>6.12$ & & & 6.244 & & & 6.300 & & & 6.291 & & \\
\hline $6{ }^{1} B_{u}^{+}$ & & & & & 6.245 & & & 6.300 & & & 6.292 & & \\
\hline $7{ }^{1} A_{g}^{-}$ & & & & & 6.388 & & & 6.792 & & & 6.789 & & \\
\hline $8{ }^{1} A_{g}^{+}$ & & & & & 6.556 & & & 6.724 & & & 6.722 & & \\
\hline $7{ }^{1} B_{u}^{-}(\|)$ & $\mathrm{m}$ & $>6.45$ & $6.9-7.2$ & & 6.731 & 0.235 & 0.004 & 7.207 & 0.240 & $1 \times 10^{-5}$ & 7.189 & 0.258 & 0.003 \\
\hline $9^{1} A_{g}^{-}$ & & & & & 6.968 & & & 7.374 & & & 7.381 & & \\
\hline $8{ }^{1} B_{u}^{-}(\perp)$ & $\mathrm{s}$ & $>6.45$ & $7.20-7.40$ & & 7.017 & 0.007 & 0.525 & 7.430 & 0.016 & 0.530 & 7.408 & 0.010 & 0.519 \\
\hline $10{ }^{1} A_{g}^{+}$ & & & & & 7.047 & & & 7.031 & & & 7.025 & & \\
\hline $9{ }^{1} B_{u}^{+}$ & & & & & 7.095 & & & 6.805 & & & 6.796 & & \\
\hline $10{ }^{1} B_{u}^{+}$ & & & & & 7.188 & & & 7.010 & & & 6.997 & & \\
\hline $11{ }^{1} B_{u}^{-}(\|)$ & $\mathrm{m}$ & & $8.1-8.7+$ & & 7.193 & $2 \times 10^{-6}$ & $5 \times 10^{-4}$ & 7.676 & $7 \times 10^{-6}$ & $3 \times 10^{-4}$ & 7.690 & $\sim 10^{-6}$ & $\sim 10^{-3}$ \\
\hline $12{ }^{1} B_{u}^{-}(\|)$ & $\mathrm{m}$ & & $8.1-8.7+$ & & 7.413 & 0.02 & 0.08 & 7.805 & 0.021 & 0.01 & 7.788 & 0.02 & 0.01 \\
\hline $17{ }^{1} B_{u}^{-}(\|)$ & $\mathrm{m}$ & & $8.1-8.7+$ & & 7.982 & $\sim 0.001$ & $\sim 0.01$ & 8.272 & $\sim 0.01$ & $\sim 0.01$ & 8.267 & $\sim 10^{-4}$ & $\sim 0.01$ \\
\hline $18^{1} B_{u}^{-}(\|)$ & $\mathrm{m}$ & & $8.1-8.7+$ & & 8.046 & $\sim 0.003$ & $\sim 0.03$ & 8.441 & $\sim 0.001$ & $\sim 0.01$ & 8.423 & $\sim 10^{-4}$ & $\sim 0.01$ \\
\hline $19{ }^{1} B_{u}^{-}(\|)$ & $\mathrm{m}$ & & $8.1-8.7+$ & & 8.348 & $\sim 0.005$ & $\sim 0.03$ & 8.730 & $\sim 0.01$ & $\sim 0.01$ & 8.704 & $\sim 10^{-4}$ & $\sim 0.01$ \\
\hline $20{ }^{1} B_{u}^{-}(\|)$ & $\mathrm{m}$ & & $8.1-8.7+$ & & 8.664 & $\sim 0.01$ & $\sim 10^{-5}$ & 8.805 & $\sim 0.01$ & $\sim 0.001$ & 8.791 & $\sim 0.01$ & $\sim 0.01$ \\
\hline \multicolumn{14}{|c|}{ Triplet spectrum } \\
\hline $1^{3} B_{u}^{+}$ & & $>2.14_{O}$ & & & 2.686 & & & 2.613 & & & 2.606 & & \\
\hline $1^{3} A_{g}^{+}$ & & & & & 4.048 & & & 3.849 & & & 3.848 & & \\
\hline $2{ }^{3} B_{u}^{+}$ & & & & & 4.314 & & & 4.274 & & & 4.258 & & \\
\hline $2{ }^{3} A_{g}^{+}$ & & & & & 4.314 & & & 4.306 & & & 4.316 & & \\
\hline $3^{3} B_{u}^{+}$ & & & & & 4.504 & & & 4.308 & & & 4.317 & & \\
\hline $3^{3} A_{g}^{+}$ & & & & & 4.665 & & & 4.556 & & & 4.556 & & \\
\hline $4{ }^{3} B_{u}^{-}$ & & & & & 4.925 & & & 5.244 & & & 5.255 & & \\
\hline $5{ }^{3} B_{u}^{+}$ & & & & & 4.928 & & & 4.795 & & & 4.770 & & \\
\hline $4{ }^{3} A_{g}^{-}$ & & & & & 4.932 & $1 \times 10^{-6}$ & 0.014 & 5.250 & $2 \times 10^{-7}$ & 0.013 & 5.257 & $1 \times 10^{-6}$ & 0.013 \\
\hline $5^{3} A_{g}^{-\frac{g}{-}}$ & $\mathrm{s}$ & $5.38-5.46$ & & & 5.659 & 0.499 & 0.011 & 6.054 & 0.489 & 0.010 & 6.050 & 0.481 & 0.013 \\
\hline $6^{3} A_{g}^{+}$ & & & & & 5.986 & & & 5.920 & & & 5.919 & & \\
\hline $6{ }^{3} B_{u}^{+}$ & & & & & 5.987 & & & 5.921 & & & 5.919 & & \\
\hline $7{ }^{3} B_{u}^{+}$ & & & & & 6.384 & & & 6.216 & & & 6.197 & & \\
\hline $7^{3} A_{g}^{+}$ & & & & & 6.420 & & & 6.223 & & & 6.216 & & \\
\hline $8^{3} B_{u}^{-}$ & & & & & 6.568 & & & 6.977 & & & 6.955 & & \\
\hline $9{ }^{3} B_{u}^{-}$ & & & & & 6.629 & & & 6.985 & & & 6.970 & & \\
\hline $8{ }^{3} A_{g}^{-}$ & & & & & 6.632 & $1 \times 10^{-6}$ & 0.011 & 6.983 & $2 \times 10^{-5}$ & 0.013 & 6.971 & $5 \times 10^{-7}$ & 0.013 \\
\hline $10^{3} B_{u}^{+}$ & & & & & 6.826 & & & 6.629 & & & 6.624 & & \\
\hline
\end{tabular}

We still find a region of parameter space giving $\Gamma=0.0 \%$. It lies near the center of the hypercube $6.9 \leqslant U$ $\leqslant 7.3 \pm 0.1 \mathrm{eV}, \quad 2.61 \leqslant t_{p} \leqslant 2.64 \pm 0.01 \mathrm{eV}, \quad 0.85 \leqslant \Delta \leqslant 1.15$ \pm 0.05 and $1.310 \leqslant \epsilon \leqslant 1.425 \pm 0.001$. The average values of the parameters giving $\Gamma=0.0$ are $U=7.163 \mathrm{eV}, \quad t_{p}$ $=2.627 \mathrm{eV}, \Delta=0.990, \epsilon=1.361$, and the center of the region is at $U=7.20 \mathrm{eV}, t_{p}=2.63 \mathrm{eV}, \Delta=0.95$, and $\epsilon=1.357$. From here on we will use this latter parameter set.

We now know the required value of $U$ to $\pm 2.8 \%$, of $t_{p}$ to $\pm 0.6 \%$, of $\Delta$ to $\pm 15 \%$ and of $\epsilon$ to $\pm 4.2 \%$. $\Delta$ only effects three hopping terms, and only via the exponent so is the least critical.

We have exactly fitted the energies, symmetries, and (for dipole allowed transitions) the polarizations of 14 states on two molecules, which amounts to fitting 39 separate pieces of information (53 if the approximate particle-hole symmetry is counted.) For a four parameter model this is very satisfactory and indicates that the model now accounts for all of the essential electronic physics of the molecules, at least below about $6-8 \mathrm{eV}$. 

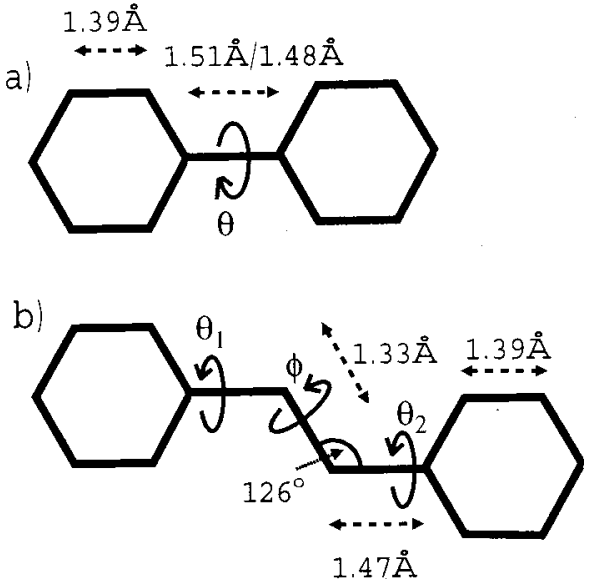

FIG. 1. Idealized geometries of (a) biphenyl (Refs. 37 and 38) (planar, $D_{2 h}$,) and (b) trans-stilbene (Refs. 39-41) (planar, $C_{2 h}$ ). For biphenyl the single bond length $r_{s}$ is $1.51 \AA$ in the condensed phases, but $1.48 \AA$ in the gas phase (Ref. 43).

The triplets and $5{ }^{1} A_{g}^{-}$of stilbene were previously omitted to limit calculation times. In principle they could now be added, together with the five known benzene states below 7 $\mathrm{eV}$, to make 21 states fitted, thus refining the fit. In practice we find that no real improvement can be made. Most of these states lie well within the experimental bounds. Those which do not show too little variation over the region of parameter space in question to be useful. We therefore take the values $U=7.20 \mathrm{eV} t_{p}=2.63 \mathrm{eV}, \Delta=0.95$, and $\epsilon=1.357$ and evaluate the full spectra. The lower states are shown in Tables I-III, but the full spectra, as high in energy as we were able to converge them, are given in the EPAPS deposit. ${ }^{16}$ For biphenyl this gives $\Gamma=0.0 \%$, for stilbene $\Gamma=0.26 \%$ (including the two additional states) and for benzene $\Gamma=0.90 \%$, giving a combined $\Gamma=0.33 \%$. The theoretical spectra agree with experiment rather well. Somewhat surprisingly, they do so right up to about $8-10 \mathrm{eV},{ }^{16}$ despite the omission of $\sigma-\pi^{*}$ transitions. They will now be discussed in more detail.

\section{B. The fitted spectra}

The fitted spectrum for biphenyl is given in columns 5 and 6 of Table II. All states fitted lie within the experimental bounds, and the oscillator strengths predicted for one photon transitions from the ground state are also in good agreement, though quantitative comparison is hard. As required, the first dipole allowed state lies above the first two dipole forbidden singlets, and now the second and third dipole allowed transitions are at the correct energies too. The vertical transitions to the first and second singlets both lie within the experimental bounds, but come in the opposite order to that observed for the OO transitions. However, the order of the vertical transitions is not certain as the possible values overlap ${ }^{10,16,18}$ and we find the separation to be only $0.025 \mathrm{eV}$-much less than the difference between the $\mathrm{OO}$ and vertical transition energies.

The higher energy dipole allowed transitions are also reasonably well described by the fitted spectrum, despite the omission of $\sigma-\pi^{*}$ type transitions, and the oscillator strength sum rule indicates there are no more one photon transitions to be observed. ${ }^{16}$ We find the three transitions between $6.5 \mathrm{eV}$ and $8.5 \mathrm{eV}$, with $\|\perp\|$ polarizations, respectively, the short one being strong, the others weak, in accordance with experiment. The first lies within experimental bounds, but is too weak. The second is $\sim 0.05 \mathrm{eV}$ too high, but has about the right oscillator strength. The last does also, but lies about $0.1 \mathrm{eV}$ too low. To get such a good description of states this high up in energy from a model that involves only $\pi$ electrons is pleasantly surprising.

The lowest lying triplet state is well described, but the second is a little $(0.3 \mathrm{eV})$ too high, and has ${ }^{3} A_{g}$ symmetry, rather than ${ }^{3} B_{2 u}$ (which lies $0.1 \mathrm{eV}$ higher again.) The calculated $T_{0} \rightarrow T_{N}$ spectrum has a strong transition to the $3{ }^{3} A_{g}^{-}$at $6.530 \mathrm{eV}$, corresponding closely to the main shoulder in the experimental spectrum. The weak $2{ }^{3} B_{3 g}^{-}$state is also quite acceptable at $5.196 \mathrm{eV} \cdot\left(1^{3} B_{3 g}^{+}\right.$at $4.532 \mathrm{eV}$ is particle-hole forbidden.) However, the first $1{ }^{3} B_{1 u}^{+} \rightarrow{ }^{3} A_{g}^{-}$ transition with a weak oscillator strength lies at $6.785 \mathrm{eV}$, which is much too high $(\sim 1.2 \mathrm{eV})$. There are no particlehole forbidden ${ }^{3} A_{g}^{+}$states in the $5.3-5.6 \mathrm{eV}$ region either, so we can only conclude that either our energy is very wrong for this one state, or that the whole of the weak band in the $T_{0} \rightarrow T_{N}$ spectrum is due to $2{ }^{3} B_{3 g}^{-}$, with perhaps some coupling to the strong $3{ }^{3} A_{g}^{-}$from nonplanarity confusing the polarization measurements. We note, however, that although we find only one strong triplet-triplet transition below $7 \mathrm{eV}$ the oscillator strength sum rule suggests that there should be more. We do indeed see some evidence for 2-3 strong transitions around $9-10 \mathrm{eV}$, mostly to states of ${ }^{3} A_{g}$ symmetry. Further transitions to ${ }^{3} B_{3 g}$ states should also exist at still higher energies. However, wave function convergence for these is not very reliable, and they lie far above the energy at which the model itself is reliable, perhaps even above the dissociation energy of the molecule. In practice, further transitions may exist, or the "lost" oscillator strength may be spread too thinly for detection.

The spectrum for stilbene (columns 5-7 of Table III) also agrees well with experiment. The lowest lying singlet transition is dipole allowed, and the first three allowed transitions are polarized correctly. In addition, our particle-hole forbidden $2{ }^{1} B_{u}^{+}$at $4.383 \mathrm{eV}$ coincides with one of the phonon side bands ${ }^{16}$ for $1{ }^{1} B_{u}^{-}$, it having long been expected that such a state should lie near here. There are also twophoton transitions at $4.386 \mathrm{eV}$ and $5.063 \mathrm{eV}$, and the particle-hole forbidden $5{ }^{1} A_{g}^{-}$state at $5.535 \mathrm{eV}, \sim 0.07 \mathrm{eV}$ too high. The experimental transition is weak, however, and could be a phonon sideband, the vertical transition being buried under the much stronger $3{ }^{1} A_{g}^{+}$, to which it is a shoulder. On the other hand, our $4{ }^{1} A_{g}^{-}$coincides with a weak shoulder in the experimental spectrum. ${ }^{20}$ The shoulder was too weak to be directly identified as an distinct transition, but our results suggest that it might be. Finally, the upturn near $6.12 \mathrm{eV}$ in the two-photon spectrum corresponds well to our $6{ }^{1} A_{g}^{+}$vertical transition at $6.244 \mathrm{eV}$. The upturn around $6.45 \mathrm{eV}$ in the one-photon absorption also indicates an $\mathrm{OO}$ transition. The argon matrix measurements ${ }^{17}$ show that it should be \| polarized, with a stronger $\perp$ transition just above. The fitted spectrum has a $\|$ transition at $6.731 \mathrm{eV}$, 
TABLE IV. Comparison of results obtained using the PPP model with (a) the "traditional" parameters $\left(t_{p}=2.4 \mathrm{eV}, U=11.26 \mathrm{eV}, \epsilon=1.0\right)$ (Refs. 4, 7), (b) the parameters optimized for $\epsilon=1.0 \quad\left(t_{p}=2.539 \mathrm{eV}\right.$, $U=10.06 \mathrm{eV}$ ), and (c) the current parameters optimised with $\epsilon \neq 0$. These are compared with results from ZINDO (Ref. 23), TD-DFT (Ref. 23), and CASPT2 (Ref. 22). State labeling is as in Table III. DFT results are given (Ref. 23) for three different exchange/correlation functionals: Slater exchange/third Vosko-Wilk-Nusair (SVWN) [equivalent to the Local Spin Density Approximation (LSDA)], Becke three-parameter hybrid exchange/Lee-Yang-Parr (B2LYP), and Becke three-parameter hybrid exchange/Perdew86 (B3P86). The $\Gamma$ error values listed are evaluated for the six transitions which are reported by all authors and for which experimental values exist.

\begin{tabular}{|c|c|c|c|c|c|c|c|c|c|}
\hline \multirow[b]{3}{*}{ State } & \multicolumn{3}{|c|}{ PPP models } & \multirow{3}{*}{ ZINDO } & \multicolumn{3}{|c|}{ DFT } & \multirow{3}{*}{ CASPT2 } & \multirow{3}{*}{ Experiment } \\
\hline & \multirow[t]{2}{*}{ Standard } & \multicolumn{2}{|c|}{ Optimized } & & \multirow[t]{2}{*}{ SVWN } & \multirow[t]{2}{*}{ B3LYP } & \multirow[t]{2}{*}{ B3P86 } & & \\
\hline & & $\epsilon=1$ & $\epsilon \neq 1$ & & & & & & \\
\hline $1{ }^{1} B_{u}^{-} \|$ & 4.25 & 4.18 & 3.99 & 3.85 & 3.70 & 3.94 & 3.95 & 4.07 & $3.98-4.04$ \\
\hline $2{ }^{1} B_{u}^{+} \perp$ & 3.91 & 4.39 & 4.38 & 4.33 & 4.03 & 4.54 & 4.55 & 3.77 & $\geqslant 4.04$ \\
\hline $2{ }^{1} A_{g}^{+}$ & 3.92 & 4.39 & 4.39 & 4.34 & 4.04 & 4.55 & 4.56 & 4.13 & $4.38-4.46$ \\
\hline $3{ }^{1} A_{g}^{+}$ & 4.57 & 5.18 & 5.06 & 5.48 & 4.48 & 5.15 & 5.17 & 4.95 & $4.99-5.07$ \\
\hline $4{ }^{1} A_{g}^{-g}$ & 5.35 & 5.30 & 5.36 & 5.60 & 4.39 & 5.08 & 5.09 & 5.30 & \\
\hline $3{ }^{1} B_{u}^{-} \perp$ & 6.03 & 6.06 & 5.41 & 5.50 & 4.46 & 5.15 & 5.17 & $5.42^{\mathrm{a}}$ & $5.35-5.43$ \\
\hline $4{ }^{1} B_{u}^{+}$ & 5.20 & 5.88 & 5.82 & & & & & 5.46 & \\
\hline $5{ }^{1} B_{u}^{-} \|$ & 5.82 & 5.80 & 6.14 & $5.61^{\mathrm{b}}$ & $5.19^{\mathrm{b}}$ & $6.35^{\mathrm{b}}$ & $6.37^{\mathrm{b}}$ & 5.95 & $6.06-6.16$ \\
\hline $7{ }^{1} B_{u}^{-} \|$ & 7.16 & 7.18 & 6.73 & 6.14 & 6.23 & 6.49 & 6.52 & & $\geqslant 6.12$ \\
\hline $1{ }^{3} B_{u}^{+}$ & 2.33 & 2.78 & 2.69 & & & & & 2.56 & $\geqslant 2.12-2.16$ \\
\hline$\Gamma$ (stilbene) & $3.6 \%$ & $1.7 \%$ & $0.0 \%$ & $1.8 \%$ & $5.0 \%$ & $1.0 \%$ & $1.0 \%$ & $1.3 \%$ & \\
\hline
\end{tabular}

${ }^{a}$ Molina et al. (Ref. 21) report two accidentally degenerate transitions at $5.42 \mathrm{eV}$ instead of just one.

${ }^{\mathrm{b}} \mathrm{Kwasniewski}$ et al. (Ref. 23) assign their ${ }^{1} B_{u}$ states at $\sim 5.1-6.4 \mathrm{eV}$ to the experimental state at $6.1 \mathrm{eV}$, as listed here. In the case of the DFT calculations, however, they have very small oscillator strengths and the next state up perhaps corresponds better to the experimental one.

oscillator strength 0.235 , followed by a $\perp$ one, oscillator strength 0.525 , at $7.017 \mathrm{eV}$. The final $\perp$ polarized band, at $8-9 \mathrm{eV}$ in the argon matrix, we find as a whole series of weak transitions, covering the correct energy range. As with the biphenyl transitions, it is pleasantly surprising to be able to describe these higher energy transitions so well with a $\pi$ electron only model. The oscillator strengths, however, are not so good-the first allowed transition being much too strong, the third too weak. This could be due to the noninclusion of phonon effects, imperfect planarity, etc.

For the lowest triplet we have only an $\mathrm{OO}$ transition available experimentally, at about $2.15 \mathrm{eV}$. We find the vertical transition at $2.686 \mathrm{eV}$, which seems a little high, but we cannot confirm this. The calculated triplet-triplet spectrum is much the same as for biphenyl; the strongest transition is the second allowed one, with a weaker one below it, which was not detected in the experiment. ${ }^{42}$ Again, the sum rule suggests more $T_{0} \rightarrow T_{n}$ transitions should exist, but we are unable to converge states high enough to find them.

The spectrum for benzene, calculated using the parameters derived for biphenyl and stilbene, is shown in column 7 of Table I. Again, most states lie within the experimental bounds, the first four singlets being described perfectly. Assignment of the high lying ${ }^{1} E_{2 g}$ states, (broader and less certain in the experimental spectra) is a little harder. Our $1^{1} E_{2 g}^{+}$corresponds to the long two photon shoulder ${ }^{28}$ around 7.3-8.4 eV. The stronger peak must then be our particlehole allowed $2{ }^{1} E_{2 g}^{-}$, lying $\sim 0.17 \mathrm{eV}$ too low. The possible experimental peak with large error bars ${ }^{28}$ at $10 \mathrm{eV}$, formally a ${ }^{1} E_{2 g}$, may be the $3{ }^{1} A_{1 g}^{+}$(allowed due to nonplanarity), or simply an artifact. We see no sign of the proposed additional ${ }^{1} E_{2 g}$ state around $5.9 \mathrm{eV}^{29}$

The predictions for the first two triplets are overestimated by about $\sim 0.2 \mathrm{eV}$, which is unfortunate. The main peak in the $T_{0} \rightarrow T_{N}$ spectrum ${ }^{28}$ fits our $2{ }^{3} E_{2 g}^{-}$, and then our particle-hole forbidden $1{ }^{3} E_{2 g}^{+}$would correspond to the peak's weak shoulder. We also find a second, much stronger $T_{0} \rightarrow T_{n}$ transition, as yet unobserved, $\sim 7 \mathrm{eV}$ above $T_{0}$. We predicts the absence of further $T_{0} \rightarrow T_{n}$ transitions after this.

To summarize, our results for biphenyl and stilbene and even benzene are in remarkably good agreement with the experimental data. The few problems mostly being restricted to the higher states, where experimental information is diffuse, and $\sigma-\pi^{*}$ transitions are may be involved. The level of accuracy attained clearly indicates that the model contains all of the fundamentally important physics.

\section{Comparison with other calculations}

Table IV compares the stilbene spectra calculated by various different techniques and with various different approximations. (The $\Gamma$ values in the table are calculated over the seven stilbene singlet states listed to allow direct comparison of all the theoretical approaches.)

Clearly, allowing $\epsilon \neq 1$ allows major improvements in the $\pi$-electron CNDO spectra. Indeed, the $\Gamma$ value obtained is better than those for ZINDO and the various ab initio calculations, although this is, of course, somewhat spurious, since the latter calculations contain many more degrees of freedom (and hence far more information) and contain no free param- 
eters. They all have qualitatively good spectra, despite the larger values of $\Gamma$. Nonetheless, it is important to note that similar levels of accuracy can be obtained using a CNDO model involving only $\pi$ electrons.

The only exception to the accuracy of the other methods is the CASPT2 value for the state we classify as $2{ }^{1} B_{u}^{+}$. CASPT2 finds it too low, unlike ZINDO and TD-DFT. We note that we could have left this state completely free during our fitting and it would still have ended up where it is. Forcing it to come down below the first dipole allowed transition as per the CASPT2 spectrum is very difficult, and messes up the rest of the spectrum.

CASPT2 spectra also exist in the literature for benzene and biphenyl, ${ }^{31,32}$ so we can also calculate a value of $\Gamma$ over the states we have fitted for all three molecules. This comes to $\Gamma=1.79 \%$, largely since CASPT 2 tends to underestimate excited state energies. The mean underestimation is $0.12 \mathrm{eV}$, and if the spectra are rigidly shifted upwards by $0.12 \mathrm{eV}$ then $\Gamma$ becomes $0.69 \%$, illustrating that the spectra are almost everywhere qualitatively good.

\section{CALCULATING THE GAS PHASE SPECTRA}

\section{A. Fitting the spectra}

In the gas phase the dipole allowed spectrum of biphenyl is known, together with the $\mathrm{OO}$ transition to the $1^{1} B_{u}^{-}$state of stilbene. This alone is not enough for a meaningful four parameter fit. However there is also a fairly complete spectrum for benzene, so we proceed by a simultaneous fit of both the benzene and biphenyl spectra. We include the first three singlet transitions of benzene, and the first two triplets. (The third was seen only using EELS, so its symmetry is not known directly.) For biphenyl the first five observed singlet transitions are included, i.e., those below $7.5 \mathrm{eV}$.

For biphenyl, easy rotation around the single bond gives very wide bell shaped peaks in the experimental spectra. The range of acceptable values for vertical transitions is thus large, even taking only the width at $90 \%$ of the maxima (Table II). The observed peaks for $2{ }^{1} B_{1 u}^{-}$and $2{ }^{1} B_{2 u}^{-}$merge completely, leaving one particularly wide peak, and the order of the states uncertain.

The mean rotation angle $\theta$ (see Fig. 1) is reported to be about $45^{\circ},{ }^{43,44}$ but the potential energy surface is rather flat, ${ }^{45}$ so we anticipate rotations of at least $15^{\circ}-20^{\circ}$ to be important. We expect that the center of each of the peaks corresponds to the $\theta=45^{\circ}$ spectrum. The peak widths may be given roughly by the difference between the spectra for $\theta=30^{\circ}$ and $\theta=60^{\circ}$, or perhaps by a wider spread of $\theta$. We first fit the spectra assuming that $\theta=45^{\circ}$, and then examine the variation with $\theta$. The single bond length, $r_{s}$, measured in the gas phase ${ }^{43}$ is $1.48 \AA$, slightly shorter than that in the crystal, due to the reduction in stearic hindrance with $\theta \neq 0$.

For stilbene, it appears ${ }^{22,46,47}$ that the isolated molecule is planar at low temperature. However, gas phase measurements ${ }^{48}$ indicate that the mean single bond rotation is about $30^{\circ}$. Calculations based upon measured phonon frequencies show that for anti-symmetric rotations $\theta_{1}=-\theta_{2}$ (see Fig. 1) the potential energy surface is very flat to around $20^{\circ}-40^{\circ}$, but only to $10^{\circ}-20^{\circ}$ for symmetric rotations. ${ }^{49}$ For the double bond rotation $\phi$ the potential energy surface is similarly flat to about $7^{\circ}$. We therefore evaluate the spectrum with both the planar geometry $\phi=\theta_{1}=-\theta_{2}=0^{\circ}$ and with $\phi=7^{\circ}, \theta_{1}=30^{\circ}=-\theta_{2}$. If the polarizability of argon is ignored, then the planar geometry, together with the gas phase parameters, should also describe the spectrum of a dilute solid solution of stilbene in argon. ${ }^{17}$

For benzene we keep the planar point group $D_{6 h}$. The space group of the biphenyl molecule with $\theta \neq 0$ is $D_{2}$, rather than the planar $D_{2 h}$. Inversion is lost and states should be labeled as ${ }^{1,3} B_{1,2,3}^{ \pm}$and ${ }^{1,3} A^{ \pm}$. For convenience, however, we keep the $D_{2 h}$ labeling, since $A_{u}$ and $B_{g}$ states cannot occur for $\pi$ electron systems anyway. The loss of inversion also means that the orthogonality of $\sigma$ and $\pi$ basis states is lost, so we anticipate mixing, at least around the single bond. Put another way, the CNDO approximation comes out slightly different again. This is most likely to effect $\Delta$. Similarly, we maintain $C_{2 h}$ labeling for stilbene, although in the nonplanar case we must calculate in $C_{2}$.

We obtain a fit at $U=8.9 \pm 0.1 \mathrm{eV}, t_{p}=2.64 \pm 0.01 \mathrm{eV}$, and $\epsilon=1.28 \pm 0.01$. The minimum of $\Gamma$ extends over a range of $\Delta$ from $-4.6 \rightarrow-3.1 \pm 0.1$, with mean relative error $\Gamma=0.046 \%$ over the 10 states used. The results given in the tables are for the midpoint, with $\Delta=-3.85$. The limits set on the acceptable energies for biphenyl at $\theta=45^{\circ}$ were rather arbitrary, however, so we expect an uncertainty in the fit of the order of maybe $10 \%$ or more. It seems prudent to claim that the "best"parameter set could have $\Gamma$ anywhere around $0.05 \%-0.06 \%$. Parameter sets meeting this criterion have $U$ ranging from $8.9 \rightarrow 9.0 \mathrm{eV}, t_{p}=2.64 \rightarrow 2.65 \mathrm{eV}, \Delta=-2.7 \rightarrow$ -5.0 , and $\epsilon=1.26 \rightarrow 1.28$.

\section{B. The fitted spectra and parameters}

The fitted spectra agree well with the experimental data. Almost all states lie exactly where they should, and the parts of the spectrum not seen or resolved experimentally support well the picture that very few qualitative changes occur between the gas and condensed phases.

In more detail, we find that, for benzene, all but the $1^{1} B_{2 u}^{+}$and $1{ }^{1} E_{1 u}^{-}$states lie within the experimental bounds. For $1{ }^{1} B_{2 u}^{+}$, the $\mathrm{OO}$ transition, lying around $4.72-4.79 \mathrm{eV}$ in most experiments, carries a lot of weight, but the strongest phonon peak is at about $4.90 \mathrm{eV}$, so our value of $4.762 \mathrm{eV}$ is a little too low. Our value for $1^{1} E_{1 u}^{-}$is low by just $0.006 \mathrm{eV}$. Looking at those states not included in the fitting, the $1{ }^{1} E_{2 g}^{+}$ state is slightly low, with a relative error of about $0.5 \%$. The third triplet $\left(3^{1} B_{2 u}^{-}\right.$, ) is slightly overestimated, with a relative error of $2 \%$. As with the condensed phases, we see no evidence of an additional ${ }^{1} E_{2 g}$ state around $5.8 \mathrm{eV}$, as proposed in the rare gas matrices. ${ }^{29}$ We therefore do not anticipate finding it at all with this model. At least the existence of such a state should be predicted by a $\pi$ electron only model so we believe that the weak intensity observed in the experiment is not due to a singlet state. It may perhaps be related to the nearby $1^{3} B_{2 u}^{-}$at $5.60 \pm 0.05 \mathrm{eV}^{50,51}$ Comparing the calculated gas phase spectrum with that for the condensed phase we note that there are no qualitative changes at all except 
above about $\sim 8.5 \mathrm{eV}$, where our model should not be reliable.

For biphenyl we find no problems with our fit at all. We do find a separation of $\sim 0.1 \mathrm{eV}$ between the second and third allowed singlet transitions, which was not resolvable in the experiments due to peak broadening. They come in the same short-long order as in the condensed phase. There are, however, a few changes compared to the condensed phase, notably two additional ${ }^{1} A_{g}$ states lie below the second dipole allowed transition, since they are less affected by screening.

For stilbene we have no experimental gas phase spectra to compare with, but changes relative to the condensed phases are fairly small. One possible exception is that the vertical transitions to $2{ }^{1} B_{u}^{+}$and to $2{ }^{1} A_{g}^{+}$lie below the first allowed transition (labeled $1{ }^{1} B_{u}^{-}$since we keep the condensed phase labeling), as they do for biphenyl, rather than above as for stilbene in the condensed phases, where it was significant for electroluminescence. The difference is tiny, however $(\sim 0.038 \mathrm{eV})$, so it lies within the uncertainty on the fit, since too few states are available for fitting. There is certainly no evidence of large scale changes in the spectrum of any of the three molecules.

For the argon matrix spectra we do not anticipate complete agreement, as we have not refitted the value of $\epsilon$, or any other parameters, assuming them to be equal to the gas values. The results are very reasonable, nonetheless, though all the energies are overestimated. This is to be expected, since the polarizability (and hence screening ability) of argon is small, but not zero as in the gas. We find all of the allowed transitions in the correct order and with roughly the correct separations between them. As with the condensed phase, the fourth experimental absorption band ${ }^{17}$ is comprised of two transitions; first a II polarized one, then a stronger $\perp$ one. For the broad fifth absorption band we again find a collection of very weak transitions rather than a single strong one. Their oscillator strengths are hard to be sure of, since they are weak and high in energy, making numerical convergence difficult. However, all of them seem to be short axis polarized, in agreement with experiment, but the sum of them is still too weak. It is also possible that with better convergence the oscillator strength of one or more may increase, since according to the sum rule, there is about 0.12 of relative oscillator strength "missing" parallel to the short axis.

The values we have obtained for $U, t_{p}$, and $\epsilon$ are physically acceptable, and will be discussed shortly. The $\Delta$ value, on the other hand, seems at first completely unphysical. However, the resulting $t_{s}$ values for biphenyl range from 2.282 to $2.514 \mathrm{eV}$ for the $0.046 \%$ fit, being $2.395 \mathrm{eV}$ at $\Delta=-3.85$. These are all perfectly reasonable. If $\theta$ were $0^{\circ}$ then $t_{s}=2.395 \mathrm{eV}$ would correspond to the acceptable $\Delta=1.504$. It seems, then, that the effect of the $\sigma-\pi$ mixing is to increase the hopping across the single bond well above that which would be expected on the basis of a pure $\pi$ electron model, partially compensating the effect of the rotation. The standard $\cos \theta$ dependence, as used here, is clearly not a good approximation for $\theta \sim 30^{\circ}-40^{\circ}$.

Turning to the other parameters, it is clear that, even in the absence of environmental screening, $U$ and $t_{p}$ are renormalized away from the values traditionally assumed. This implies that the effective screening by the $\sigma$ electrons is slow or intermediate in rate. Indeed, almost all of the renormalization of the parameters has already occurred, even here in the gas phase; $U=8.9 \mathrm{eV}$, compared to $7.2 \mathrm{eV}$ for the condensed phases, or to $11.13 \mathrm{eV}$ anticipated in the absence of screening; $t_{p}=2.64 \mathrm{eV}$ for both condensed and gas phases, compared to the $2.4 \mathrm{eV}$ anticipated; $\epsilon=1.28$ compared to $1.31-$ 1.43 for the condensed phase, but 1.00 in the absence of any screening (aside from the screening from the $\pi$ electrons themselves).

So, the spectra of all three molecules are here predicted to have essentially the same structure and ordering in the gas, argon matrix, and hydrocarbon matrix condensed phases, despite the solvent shifts, which do effect states of different symmetry to slightly differing extents. The ordering of the dipole allowed transitions remains unchanged, despite the markedly different screening abilities of the different environments, and almost all of the parameter renormalization is already present in the gas phase. Hence the most important screening interactions are internal to the molecules themselves.

\section{The value of $U$}

As discussed in Sec. III, the value of $U$ is normally taken as $11.13 \mathrm{eV}$, though the error bar is probably large. The $U$ parameter occurring within our model is not quite equivalent to it, since it was renormalized while reworking the CNDO approximation. The reduction of $U$ is, in part, a consequence of allowing for differential overlap terms which are otherwise and elsewhere omitted.

It is instructive, however, to try to estimate an effective value $U^{\text {eff }}=(I-A)$ within our parameterization of the model, to compare directly to the traditional value. (This we do here for the gas phase case, but the condensed phases would come out similarly.) This cannot be done completely, since the atomic core potential does not occur explicitly in our model. If we make the assumption that it is identical on all atoms, irrespective of their occupancy, then it cancels out. If we then calculate the total electronic energy in the ground state of benzene with five electrons, and subtract it from that with seven electrons we get an estimate for $U^{\text {eff }}$. (At CCI we are restricted to benzene by the loss of the particle-hole and spin-flip symmetries.) We find $U^{\mathrm{eff}}=10.0 \mathrm{eV}$, exactly halfway between the $8.9 \mathrm{eV}$ value of $U$ itself and the traditional value of $11.13 \mathrm{eV}$. Referring to Sec. III, this estimate does not include the difference in electronic relaxation between the process (0) (leaving a hole behind) and the combination $(1)+(2)$ (ignoring the hole). The resulting error in $U^{\text {eff }}$ should be on the order of the screening energy contribution to $(I-A)$. The environmental screening is zero. With our present code we cannot directly estimate the $\pi$ electron screening contribution, since all orders of CI are automatically included. However, we can estimate it for the $\sigma$ electron screening, simply by setting $\epsilon=1.00$. We find the improved estimate $U^{\mathrm{eff}}=11.24 \mathrm{eV}$. This overestimates the correction from the $\sigma$ screening, but since we ignore the corrections from the $\pi$ screening the estimate $U^{\mathrm{eff}}=(I-A)$ $=11.0 \pm 1.0 \mathrm{eV}$ is reasonable. It is in agreement with the tra- 
TABLE V. Results of biphenyl calculations: energies $\left(E_{n}\right.$, in eV) and oscillator strengths $\left(O_{n}\right)$ of dipole allowed transitions as a function of $\theta$. (Oscillator strength are for transitions from the ground state.)

\begin{tabular}{|c|c|c|c|c|c|c|}
\hline \multirow[b]{2}{*}{ State } & \multicolumn{2}{|c|}{$\theta=0^{\circ}$} & \multicolumn{2}{|c|}{$\theta=45^{\circ}$} & \multicolumn{2}{|c|}{$\theta=90^{\circ}$} \\
\hline & $E_{n}$ & $O_{n}$ & $E_{n}$ & $O_{n}$ & $E_{n}$ & $O_{n}$ \\
\hline $2{ }^{1} B_{2 u}^{+}$ & 4.494 & 0.000 & 4.759 & 0.000 & 4.761 & 0.000 \\
\hline $1^{1} B_{1 u}^{-}$ & 5.109 & 0.399 & 5.123 & 0.398 & 6.264 & 0.025 \\
\hline $2{ }^{1} B_{2 u}^{-}$ & 6.394 & 0.517 & 6.368 & 0.450 & 6.921 & 0.491 \\
\hline $2{ }^{1} B_{1 u}^{-}$ & 6.496 & 0.501 & 6.502 & 0.501 & 6.649 & 0.954 \\
\hline $3^{1} B_{1 u}^{-}$ & 7.227 & 0.001 & 7.228 & $5 \times 10^{-4}$ & 7.071 & 0.000 \\
\hline $3{ }^{1} B_{2 u}^{-}$ & 7.757 & 0.431 & 7.735 & 0.363 & 7.300 & 0.000 \\
\hline $4{ }^{1} B_{1 u}^{-}$ & 8.162 & 0.071 & 8.158 & 0.075 & 7.474 & 0.000 \\
\hline
\end{tabular}

ditional value $U=11.13 \mathrm{eV}$, and hence our parameterization is also in agreement with the original experimental data which led to it.

\section{Peak widths in the spectrum for biphenyl}

Since the apparent $\sigma-\pi$ mixing so strongly affects the value of $t_{s}$ it is not clear how to calculate the spectra for $\theta=30^{\circ}$ and $\theta=60^{\circ}$. Instead, we calculate the spectra for $\theta=90^{\circ}$, where $t_{s}$ should still be zero, and $\theta=0^{\circ}$ where the mixing should be zero. We use the value $\Delta=0.95$ from the condensed phase. The resulting spectra are shown in of Table V. Taking the strong variation in some of the oscillator strengths into account we would anticipate the existence of five peaks in the spectrum, covering $5.1-\sim 5.8 \mathrm{eV}, 6.4-6.9$ $\mathrm{eV}, 7.1-7.2 \mathrm{eV}, \sim 7.4-7.7 \mathrm{eV}$, and $\sim 7.8-8.2 \mathrm{eV}$, with perhaps a weak shoulder from the particle-hole forbidden $1^{1} B_{2 u}^{+}$around 4.5-4.8 eV. This compares well with the peaks actually observed. The last is too low in the calculation, but at $\sim 8 \mathrm{eV}$ errors are to be expected. Residual phonon broadening can account for the rest of the peak widths. Subject to the uncertainties due to the $\sigma-\pi$ mixing, the peak widths thus seem reasonably well accounted for.

\section{CONCLUSIONS}

By comparing various experiments we first noted that the differences in solvent/crystal shifts in the spectra of the three molecules benzene, biphenyl, and trans-stilbene in different hydrocarbon based condensed phases are similar to the uncertainty in the vertical transition energies for most states in the individual spectra. It is then reasonable to compile them into a single composite spectrum, with error bars set by the spread and width in the measured energies.

We then proposed a semiempirical CNDO model, of the PPP type, for calculating the spectra, with four free parameters which we fitted using CCI calculations for the three molecules. For the hydrocarbon matrix condensed phases we succeeded in simultaneously fitting 21 states, including complete symmetry assignments and (for dipole allowed transitions) polarizations for the condensed phase spectra, with a further 10 or so other states at higher energies also well described. We obtained the fitted values $U=7.20 \mathrm{eV}, t_{p}$ $=2.63 \mathrm{eV}, \Delta=0.95$, and $\epsilon=1.357$. This gave an average relative error over the 21 states of $\Gamma=0.33 \%$ (total error 0.66 $\mathrm{eV}$ ) compared to $\Gamma=1.49 \%$ (total error $3.58 \mathrm{eV}$ ) for the best previous CNDO calculations. ${ }^{5,6}$ It also compares very favorably to ZINDO, ab initio TD-DFT, and CASPT2.

More specifically, we were able to fit singlet spectra virtually perfectly, and to higher energies than nominally expected from a $\pi$ electron only model: up to $8-10 \mathrm{eV}$, which is well into the energy range at which $\sigma-\pi^{*}$ type transitions and ionization should be important, and is perhaps surprising. For triplets, errors were sometimes slightly larger, maybe a few percent for an individual state. For oscillator strengths, the polarization directions and the general trends in the magnitudes were correct. However, some were not perfect quantitatively. This is probably due partly to the omission of $\pi-\sigma^{*}$ type transitions, but mostly to our noninclusion of phonon effects.

For the gas phase spectra, we have fitted nine states of benzene and biphenyl, with a mean relative error on the order of $0.05 \%$, limited not by our fitting, but by the width of the experimental peaks. This gave the parameters $U$ $=8.9 \mathrm{eV}, t_{p}=2.64 \mathrm{eV}$ and $\Delta=-3.85$ and $\epsilon=1.28$. For stilbene, calculated results agreed well with the absorption spectrum in a solid argon matrix.

To obtain this kind of accuracy suggests that we have, in effect, found that which was missing from previous $\pi$-electron only CNDO approaches. What we have done is to use a slightly more complex scheme for the empirical adjustment of the diagonal overlap elements, taking better account of the neglected differential overlap terms. This amounts to renormalizing both $U$ and $t_{p}$ [such that our $U$ is no longer equivalent to $(I-A)]$ and introducing a dielectric constant $\epsilon$. Nonetheless, an estimate of $U^{\mathrm{eff}}=(I-A)$ in the gas phase was in keeping with the common value of $11.13 \mathrm{eV}$.

This all shows that the screening effects are indeed essential for an accurate description of the spectra of these molecules, as evidenced by the value of $\epsilon$ being significantly greater than 1.0. However, we found that most of the significant features of the spectra, and almost all of the parameter renormalization, are already present even in the gas phase. Since these effects are already present in the gas phase they cannot be due to external environmental factors, as suggested elsewhere. ${ }^{15}$ Environmental screening certainly has an important role, via solvent shifts, etc., which can lead to some reordering of states, which in turn may, of course, be physically significant in some cases. However, this is on the order of tenths of $\mathrm{eV}$. The crossing over of these states when we do and do not include the dielectric constant in the calculation is of the order of eV. Since the dielectric constant and parameter renormalization are needed even to reproduce the gas and argon matrix spectra the principle screening must be an internal property of the molecules themselves. Physically, it amounts to a description of the slow or intermediate rate screening of the $\pi-\pi$ electron interactions by the $\sigma$ electrons. This feature of such molecules is automatically included in most $a b$ initio calculations, and in also handled by some CNDO and INDO calculations which explicitly include the $\sigma$ electrons. However, we have shown that, to a reasonable extent, the effect of $\sigma$ electron screening need be included to mean field order only, as parameter renormalizing factors in the model. Hence it can be included in a $\pi$ electron only CNDO model, with its far smaller Hilbert space and 
faster computation times, and/or larger system sizes.

Our calculations were done at Complete Configuration Interaction level. In order to fully make use of this reduction in Hilbert space size an examination is now required of the level of CI needed to describe the $\pi$ electron correlations and screening adequately within our framework. On the other hand, the new parameterization can be applied straight away to improve current density matrix renormalization group (DMRG) calculations on the related polymers.

To conclude, we have shown that for accurate theoretical descriptions of the optical spectra and electronic states of $\pi$ conjugated molecules we need to include the effective screening from the $\sigma$ electrons. Screening from the environment, while certainly present, and in some cases perhaps significant, is nonetheless much less important from the point of view of practical calculations to understand the photophysics of these and related molecules.

\section{ACKNOWLEDGMENTS}

The authors acknowledge support from the EPSRC (GR/ K86343,) and C.W.M.C. would like to thank P. A. Lane for helpful discussions.

${ }^{1}$ D. L. Beveridge and H. H. Jaffé, J. Am. Chem. Soc. 87, 5340 (1965); P. Swiderek, M. Michaud, G. Hohlneicher, and L. Sanche, Chem. Phys. Lett. 187, 583 (1991).

${ }^{2}$ F. Momicchioli, I. Baraldi, and M. C. Bruni, Chem. Phys. 82, 229 (1983).

${ }^{3}$ Z. G. Soos, S. Ramasesha, D. S. Galvãno, and S. Etemad, Phys. Rev. B 47, 1742 (1993).

${ }^{4}$ S. Ramasesha, I. D. L. Albert, and B. Sinha, Mol. Phys. 72, 537 (1991).

${ }^{5}$ R. Bursill, W. Barford, and C. W. M. Castleton, Chem. Phys. Lett. 294, 305 (1998).

${ }^{6}$ C. W. M. Castleton and W. Barford, Synth. Met. 101, 520 (1999).

${ }^{7}$ R. Pariser and R. Parr, J. Chem. Phys. 21, 466 (1953); 21, 767 (1953).

${ }^{8}$ K. Ohno, Theor. Chim. Acta 2, 219 (1964).

${ }^{9}$ G. Orlandi, P. Palmieri, and G. Poggi, J. Am. Chem. Soc. 101, 3492 (1979); F. Negri and M. Z. Zgierski, J. Chem. Phys. 97, 7124 (1992).

${ }^{10}$ J. Sagiv, A. Yogev, and Y. Mazur, J. Am. Chem. Soc. 99, 6861 (1977).

${ }^{11}$ B. Dick and G. Hohlneicher, Chem. Phys. 94, 131 (1985).

${ }^{12}$ K. Schulten, I. Ohmine, and M. Karplus, J. Chem. Phys. 64, 4422 (1976).

${ }^{13}$ E. Moore, B. Gherman, and D. Yaron, J. Chem. Phys. 106, 4216 (1997).

${ }^{14}$ E. Moore and D. Yaron, J. Chem. Phys. 109, 6147 (1998).

${ }^{15}$ M. Chandross, S. Mazumdar, M. Liess, P. A. Lane, Z. V. Vardeny, M. Hamaguchi, and K. Yoshino, Phys. Rev. B 55, 1486 (1997).

${ }^{16}$ See EPAPS Document No. E-JCPSA6-117-303230 for complete spectra. Namely, all available experimental spectra, with references, and all converged PPP model states (up to $10-15 \mathrm{eV}$ ). This document may be retrieved via the EPAPS homepage (http://www.aip.org/pubservs/ epaps.html) or from ftp.aip.org in the directory /epaps/. See the EPAPS homepage for more information.

${ }^{17}$ M. S. Gudipati, M. Maus, J. Daverkausen, and G. Hohlneicher, Chem. Phys. 192, 37 (1995).
${ }^{18}$ B. Dick and G. Hohlneicher, Chem. Phys. 94, 131 (1985).

${ }^{19}$ R. P. Drucker and W. M. McClain, J. Chem. Phys. 61, 2609 (1974).

${ }^{20}$ G. Hohleneicher and B. Dick, J. Photochem. 27, 215 (1984).

${ }^{21}$ B. O. Roos, K. Andersson, M. P. Fülscher, L. Serrano-Andres, K. Pierloot, M. Merchan, and V. Molina, J. Mol. Struct. 388, 257 (1996).

${ }^{22}$ S. P. Kwasniewski, M. S. Deleuze, and J. P. Francois, Int. J. Quantum Chem. 80, 672 (2000).

${ }^{23}$ Y. H. Meyer, R. Astier, and J. M. Leclercq, Chem. Phys. Lett. 4, 587 (1970).

${ }^{24}$ D. Lavalette and C. Tetreau, Chem. Phys. Lett. 29, 204 (1974).

${ }^{25}$ R. Astier and Y. H. Meyer, Chem. Phys. Lett. 3, 399 (1969).

${ }^{26}$ R. L. Whetton, K-J. Fu, and E. R. Grant, J. Chem. Phys. 79, 2626 (1983).

${ }^{27}$ S. G. Grubb, C. E. Otis, R. L. Whetton, E. R. Grant, and A. C. Albrecht, J. Chem. Phys. 82, 1135 (1985).

${ }^{28}$ N. Nakashima, M. Sumitani, I. Ohmine, and K. Yoshihasa, J. Chem. Phys. 72, 2226 (1980).

${ }^{29}$ G. C. Morris and J. G. Angur, J. Mol. Spectrosc. 45, 271 (1973).

${ }^{30}$ J. Obrzut and F. E. Karasz, J. Chem. Phys. 87, 2349 (1987); I. V. Sokolova, N. Yu. Vasil'eva, Ya. O. Vylegzhanina, and G. V. Maier, Russ. Phys. J. 36, 882 (1993).

${ }^{31}$ V. Molina, M. Merchan, and B. O. Roos, J. Phys. Chem. A 101, 3478 (1997).

32 J. Lorentxon, P-Å. Malmqvist, M. P. Fülscher, and B. O. Roos, Theor. Chim. Acta 91, 91 (1995).

${ }^{33}$ J. Hinze and H. H. Jaffé, J. Am. Chem. Soc. 84, 540 (1962).

${ }^{34}$ D. Baeriswyl, D. K. Cambell, and S. Mazumdar, in Conjugated Polymers, edited by H. Kiess (Springer-Verlag, Berlin, 1992).

${ }^{35}$ F. Negri, G. Orlandi, and F. Zerbetto, J. Phys. Chem. 93, 5124 (1989).

${ }^{36}$ L. Salem, Molecular Orbital Theory of Conjugated Systems (Benjamin, New York, 1966).

${ }^{37}$ A. Hargreaves and S. Hasan Rizvi, Acta Crystallogr. 15, 365 (1962).

${ }^{38}$ G-P. Charbonneau and Y. Delugeard, Acta Crystallogr., Sect. B: Struct. Crystallogr. Cryst. Chem. B32, 1420 (1976); B33, 1566 (1977).

${ }^{39}$ C. J. Finder, M. G. Newton, and N. L. Allinger, Acta Crystallogr., Sect. B: Struct. Crystallogr. Cryst. Chem. B30, 411 (1979).

${ }^{40}$ J. Bernstein, Acta Crystallogr., Sect. B: Struct. Crystallogr. Cryst. Chem. B31, 1268 (1975).

${ }^{41}$ J. A. Bouwstra, A. Schouten, and J. Kroon, Acta Crystallogr., Sect. C: Cryst. Struct. Commun. C40, 428 (1984).

${ }^{42}$ W. G. Herkstroeter and D. S. McClure, J. Am. Chem. Soc. 90, 4522 (1968).

${ }^{43}$ R. M. Barret and D. Steele, J. Mol. Struct. 11, 105 (1972).

${ }^{44}$ A. Almenningen, O. Bastiansen, L. Fernholt, B. N. Cyvin, S. J. Cyvin, and S. J. Samdal, J. Mol. Struct. 128, 59 (1985).

${ }^{45}$ S. Tsuzuki and K. Tanabe, J. Phys. Chem. 95, 139 (1991).

${ }^{46}$ L. H. Spangler, R. van Zee, and T. S. Zwier, J. Phys. Chem. 91, 2782 (1987).

${ }^{47}$ B. B. Champagne, J. F. Pfanstiel, D. F. Plusquellic, D. W. Pratt, W. M. van Herpen, and W. L. Meerts, J. Phys. Chem. 94, 6 (1990).

${ }^{48}$ M. Traetteberg, E. B. Frantsen, F. C. Mijlhoff, and A. Hoekstra, J. Mol. Struct. 26, 57 (1975).

${ }^{49}$ W-Y. Chiang and J. Laane, J. Chem. Phys. 100, 8755 (1994).

${ }^{50}$ J. P. Doering, J. Chem. Phys. 51, 2866 (1969).

${ }^{51}$ R. P. Frueholz, W. M. Flicker, O. A. Mosher, and A. Kupperman, J. Chem. Phys. 70, 3057 (1979). 